\title{
The relationship of femoral neck shaft angle and adiposity to greater trochanteric pain syndrome in women. A case control morphology and anthropometric study
}

\author{
Fearon AM, ${ }^{1,2}$ Stephens $\mathrm{S}_{1}{ }^{2}$ Cook $\mathrm{JL}^{3}{ }^{3}$ Smith PN, ${ }^{1,2}$ Neeman $T,{ }^{4}$ Cormick $\mathrm{W}^{5}$ \\ Scarvell JM ${ }^{2}$
}

- Additional tables are published online only. To view the files please visit the journal online (http://bjsm.bmj.com/ content/46/12.toc)

${ }^{1}$ Department of Medicine Biology and the Environment, Australian National University, Canberra, Australian Capital Territory, Australia

2Department of Surgery, The Trauma and Orthopaedic Research Unit, Canberra, Australian Capital Territory, Australia

${ }^{3}$ Primary Health Care, Monash University, Melbourne,

Victoria, Australia

${ }^{4}$ Statistical Consulting Unit, Australian National University, Acton, Australian Capital

Territory, Australia

${ }^{5}$ Canberra Specialist

Ultrasound, Deakin West, Australian Capital Territory, Australia

\section{Correspondence to}

Fearon AM,

Centre for Hip Health and

Mobility, 7th Floor, 2635 Laurel

St Robert H.N. Ho Research

Centre Vancouver, BC, Canada

V5Z $1 \mathrm{M} 9$

angie.fearon@hiphealth.ca

Received 9 November 2011 Accepted 12 March 2012

Published Online First

30 April 2012

\author{
ABSTRACT \\ Objective To evaluate if pelvic or hip width predis- \\ posed women to developing greater trochanteric pain \\ syndrome (GTPS).
}

Design Prospective case control study.

Participants Four groups were included in the study: those gluteal tendon reconstructions ( $n=31, G T R)$, those with conservatively managed GTPS $(n=29)$, those with hip osteoarthritis $(n=20,0 A)$ and 22 asymptomatic participants (ASC).

Methods Anterior-posterior pelvic x-rays were evaluated for femoral neck shaft angle; acetabular index, and width at the lateral acetabulum, and the superior and lateral aspects of the greater trochanter. Body mass index, and waist, hip and greater trochanter girth were measured. Data were analysed using a one-way analysis of variance (ANOVA; posthoc Scheffe analysis), then multivariate analysis.

Results The GTR group had a lower femoral neck shaft angle than the other groups $(p=0.007)$. The $O R$ $(95 \% \mathrm{CI})$ of having a neck shaft angle of less than $134^{\circ}$ relative to the $A S C$ group: $\mathrm{GTR}=3.33$ (1.26 to 8.85$)$; $\mathrm{GTPS}=1.4$ (0.52 to 3.75$) ; 0 A=0.85$ (0.28 to 2.61). The OR of GTR relative to GTPS was 2.4 (1.01 to 5.6). No group difference was found for acetabular or greater trochanter width. Greater trochanter girth produced the only anthropometric group difference (mean $(95 \% \mathrm{CI})$ in $\mathrm{cm}) \mathrm{GTR}=103.8$ (100.3 to 107.3), GTPS =105.9 (100.2 to 111.6), $\mathrm{OA}=100.3$ (97.7 to 103.9), $\mathrm{ASC}=99.1$ (94.7 to 103.5), (ANOVA: $p=0.036$ ). Multivariate analysis confirmed adiposity is associated with GTPS.

Conclusion A lower neck shaft angle is a risk factor for, and adiposity is associated with, GTPS in women.

\section{INTRODUCTION}

Greater trochanteric pain syndrome (GTPS) is thought to be a degenerative condition of the gluteal tendons and bursa ${ }^{1} 2$ for which few risk factors have been identified. Tendinopathies occur in both sporting and sedentary populations ${ }^{3}$ indicating a multifactorial aetiology. ${ }^{4-6}$ These factors include intrinsic and extrinsic risk factors. ${ }^{5}$ Identifying risk factors for GTPS should aid in understanding the aetiology of, and direct treatment for, GTPS.

Intrinsic risk factors previously identified for tendinopathies such as patellar and Achilles tendinopathy include genetic and biomechanical factors. ${ }^{78}$ Intrinsic factors associated with GTPS are sex and age, ${ }^{9} 10$ with older women at greater risk of developing GTPS. Other possible intrinsic factors are adiposity and biomechanics. Elevated adiposity has been proposed as an under recognised risk factor for all tendinopathies ${ }^{11}$ suggesting a systemic mechanism is involved rather than mechanical overload. ${ }^{6}$ Peripheral adiposity, (gynoid distribution), appears to be a risk factor for developing tendinopathy in women. ${ }^{12}$ To date, the level of adiposity in GTPS has only been reported in terms of body mass index (BMI), with values ranging from $22.1 \mathrm{kgm}^{-213}$ to $27.3 \mathrm{kgm}^{-2}{ }^{14}$ BMI measures the relationship between height and weight but does not indicate fat distribution, a key factor in determining the systemic effects of increased adiposity. ${ }^{15}$ As there is some evidence to suggest increased adiposity is a risk factor for developing tendinopathy in women, the relationship of adiposity and GTPS deserves further investigation.

Other intrinsic risk factors for developing GTPS that have been reported in review papers and textbooks (in runners) include leg length discrepancy $^{16}$ and pelvic width. ${ }^{16-19}$ However, leg length discrepancy has not been found to be associated with GTPS in people with primary GTPS ${ }^{10}$ or in people who develop GTPS post-total hip arthroplasty. ${ }^{20}$ In addition, leg length discrepancy has been reported to be lower in those with GTPS than the population prevalence. ${ }^{21} 22$ The evidence of pelvic width as a risk factor is limited to level V evidence, ${ }^{1617}$ with the authors failing to differentiate between the pelvic, hip or greater trochanter width. As GTPS is more common in women, and women have wider pelves than men, ${ }^{23}$ the research question of whether a wider pelvis is an intrinsic risk factor for GTPS should be addressed.

Pathological compression of a tendon is thought to contribute to the development of tendinopathy. ${ }^{24-26}$ The angle that the tendon approaches the enthesis and the size of the bony pulley before the tendon insertion affects the strain and compression forces on the tendon. In the case of the gluteus medius and minimus tendons, this angle is in part determined by the neck shaft angle of the femur. In addition, greater hip width at the level of the greater trochanters would result in the gluteal tendons wrapping over a greater proportion of the greater trochanter, leading to an increased area of compression. ${ }^{27}$ Therefore, the degree of coxa vara and the pelvic width at the level of the 
greater trochanter may be a predisposing factor for developing GTPS. ${ }^{24-26}$

The aims of this study were to evaluate if the width of the pelvis or femoral neck shaft angle constituted independent risk factors, or if in combination of these constituted a risk factor for developing GTPS. A further aim was to determine if increased adiposity and the distribution pattern of the adiposity was associated with GTPS.

\section{PARTICIPANTS AND METHODS}

\section{Participants}

Four groups of participants were recruited; those with GTPS, gluteal tendon reconstruction (GTR; surgical management of GTPS), hip osteoarthritis (OA) and an age and sex matched (to the GTPS group) control group (ASC). Participants were recruited through private healthcare providers and viva voce. All participants had to be older than 18 years of age and able to communicate in English. Participants were excluded if they had the following: systemic inflammatory disease, for example, rheumatoid arthritis; lumbar spine nerve root signs; a history of lumbar spine or ipsilateral hip joint surgery; osteogenic disease, for example, Paget's disease, or neoplastic disease. Due to sex-linked pelvic morphology, men were excluded from this study. ${ }^{23}$

Hip OA participants were diagnosed according to Altman $1991^{28}$ and were scheduled for hip arthroplasty surgery. Diagnostic criteria for GTPS was a history of lateral hip pain, ${ }^{22} 29$ pain on palpation of the greater trochanter and at least one of lateral hip pain with lying on the ipsilateral side, weight bearing activities or sitting. ${ }^{29} 30$ In addition to the above criteria for GTPS, participants in the GTR group had evidence of tendon tearing on MRI or ultrasound imaging, with symptoms refractory to ultrasound-guided corticosteroid injection and physiotherapy. Control participants had to be asymptomatic for hip joint and tendon pathology. Participants in the GTPS, GTR and ASC groups had no evidence of hip OA. ${ }^{28}$ Participants in the ASC and OA groups had no past or current history of GTPS. Clinical status was confirmed by examination using the above criteria (AF). Hip joint status was confirmed via x-ray or MRI.

Ethical approval for this study was provided by the Australian National University, ACT Health, Calvary HealthCare and Deakin University human research ethics committees. All participants provided informed consent.

\section{METHODS}

The study was undertaken between February 2004 and November 2009. Two hundred and twenty potential participants were contacted. Within the GTPS group, 40 people were contacted, 10 were excluded, and one withdrew $(n=29)$. Within the GTR group, 35 potential participants were contacted, and four were excluded $(n=31)$. Eleven were assessed preoperatively; the remainder were assessed an average of 22 months postoperatively. Details of the latter group have been previously reported. ${ }^{1}$ Within the ASC group, 40 people were contacted, 18 were excluded $(n=22)$. Within the OA group, 126 people were contacted, 23 declined, 73 were excluded $(\mathrm{n}=20)$.

Participants had a standardised anterior to posterior x-ray in supine, centred on the pubis. The x-rays were digitised and deidentified. Pelvic morphology was assessed by measures of lateral pelvic dimensions and the acetabular index using Intellicad 2000 for Microsoft windows (IntelliCAD technology consortium, Visio Corporation). Lateral measures were

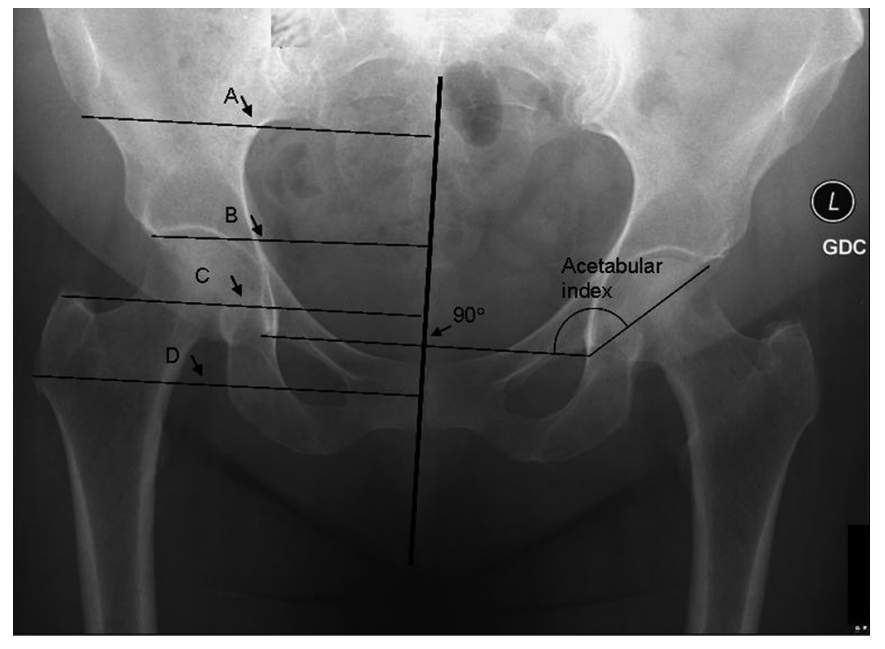

Figure 1 Schematic illustration of the lines used to measure the width of the pelvis and greater trochanters, and the acetabular index. The midline was defined as the line perpendicular and bisecting the line that joined to two tear drops. (A) The width from the midline to the anterior inferior iliac spine - the reference width. (B) The width from the midline to the most lateral aspect of the acetabulum. (C) The width from the midline to the most superior point of the greater trochanter. (D) The width from the midline to the most lateral aspect of the greater trochanter.

taken from the midline - a perpendicular line that bisected the distance between the distal border of the acetabular tear drops (ML) to the anterior inferior iliac spine (AIIS) (A); to the lateral rim of the acetabulum (B); to the most superior aspect of the greater trochanter $(C)$; and to the most lateral aspect of the greater trochanter (D) (figure 1). Ratio distances in relation to A were calculated for linear measures. The acetabular index was measured according to Sharp's method ${ }^{31}$ (figure 1). The femoral neck shaft angle was measured using a six-inch goniometer (http://www.physiosupplies.com.au 15 May 2011) on the digital x-ray, aligning the goniometer arms along the shaft and neck of the femur. Measurements were undertaken by one of two assessors (AF, SS). To test the reliability of measurements, each rater measured an identical set of 20 deidentified $\mathrm{x}$-rays twice, 1 week apart.

\section{Physical examination}

Ninety nine participants had standard waist and hip girth measurements taken (AF). In standing, using a non-stretch tape measure, the waist measure was taken on light expiration at the narrowest point, ${ }^{32}$ (or the mid point between the ribs and the iliac crest when the narrowest point was not identifiable). The hip measure was taken at the largest elevation about the buttocks; ${ }^{32} 33$ and at the greater trochanter, across both greater trochanters with the tape measure parallel to the ground. Height and weight were measured with the participants in light clothing and no shoes. Height was measured using a wall-mounted stadiometer (Menton Education Centre, Moorabbin, http://mentone-educational.com.au). Weight was measured on flat medical quality scales (Hills scale service, Baulkham Hills, NSW. http://www.hillscales.com.au).

\section{Data analysis}

Statistical analyses were performed with Stata IC 10.1 (Statacorp, USA). Reliability was assessed using an intraclass correlation co-efficient (ICC). Between group, differences were 
compared using a one-way analysis of variance (ANOVA) with posthoc Scheffe analysis. Linear models were used to test group differences between the affected and unaffected lower limbs. In cases of bilateral symptoms, the more symptomatic lower limb was designated the affected side. Analysis by side led to the same conclusions regarding group differences, thus the mean neck of femur neck shaft angle and acetabular index from both sides is reported. Between group differences were calculated for the neck shaft angle and acetabular index using a linear mixed-model analysis. $p$ Values of $<0.05$ were considered significant. Using a posthoc cut off of $134^{\circ}$ for neck shaft angle, logistic regression was used to assess group differences in the prevalence of a neck shaft angle less than $134^{\circ}, \mathrm{OR}$ (95\% CI) relative to the asymptomatic group are reported. A canonical linear discriminant analysis (CDA) was undertaken to explore group differences in relation to multiple variables. All variables were included in the CDA analysis.

\section{RESULTS}

\section{Participants}

The OA participants were the oldest cohort, with a mean (SD) age of 62 (13.3) years. In contrast, the mean ages for the ASC, GTPS and GTR groups were 51.6, 52.2 and 55.7 years, respectively (table 1).

Table 1 Demographic data and x-ray and anthropometric data availability by group

\begin{tabular}{lllll}
\hline & ASC (n=22) & GTPS (n=29) & GTR (n=31) & OA (n=20) \\
\hline Age (years): mean (SD)* & $51.6(10.19)$ & $52.2(12.85)$ & $55.7(9.13)$ & $62.0(13.28)$ \\
$\begin{array}{l}\text { Duration of symptom: } \\
\text { mean (SD, months) } \dagger\end{array}$ & 0 & $61.9(60.2)$ & $44.4(37.4)$ & $40.3(43.9)$ \\
X-ray reports & $20 / 22$ & $28 / 29$ & $31 / 31$ & $20 / 20$ \\
X-ray images $\ddagger$ & $20 / 22$ & $28 / 29$ & $21 / 31$ & $20 / 20$ \\
Anthropometric data & $22 / 22$ & $28 / 29$ & $30 / 31$ & $20 / 20$
\end{tabular}

ACS, asymptomatic control group; GTPS, greater trochanteric pain syndrome group; GTR, gluteal tendon reconstruction group; OA, hip osteoarthritis group. ${ }^{*} \mathrm{p}<0.05$, one way analysis of variance.

†No group difference between the symptomatic groups. The GTR group data are for the 11 prospectively enrolled participants. The remaining GTR participants were evaluated a mean of 22 months postoperatively.

¥Missing $\mathrm{x}$-rays: one ASC and one GTPS participant did not consent to $\mathrm{x}$-ray; $\mathrm{X}$-ray imaging was missing for one ASC and 10 GTR participants. X-ray or MRI reports were available for all participants confirming the status of the joint.
Inter and intratester reliability

Good to excellent intra and intertester reliability was demonstrated for all imaging measures, greater than 0.70 being considered good. ${ }^{34} 35$ The intrarater ICC ranged from $(95 \%$ $\mathrm{CI})$ of $0.72(0.62$ to 0.80$)$ to $1.0(1.0$ to 1.0$)$ and the interrater ICC range ( $95 \% \mathrm{CI}$ ) of 0.77 (0.72 to 0.82 ) to 0.99 (0.98 to 1.0$)$ (table S1, table S2).

\section{Bony morphology}

The neck shaft angle was lower in the GTR participants than in any other group (table 2). No difference was demonstrated between the GTPS, OA or ASC groups. The OR (95\% CI) of having a neck shaft angle of less than $134^{\circ}$, relative to the ASC group was highest for the GTR group at 3.33 (1.26 to 8.85); 1.4 (0.52 to 3.75) for the GTPS; and 0.85 (0.28 to 2.61) for the OA group. The OR of GTR relative to GTPS was 2.4 (1.01 to 5.6). No group difference was found for the acetabular index, any measure of pelvic width, or the distance from the superior greater trochanter to the lateral greater trochanter (table 2).

\section{Anthropometric measurements}

There was statistically significant difference across the groups in greater tronchanter girth measurements (ANOVA: $p=0.036$, table 3). GTPS group had the highest, and the ASC group had the lowest, greater trochanter girth measurements. However, posthoc analysis failed to demonstrate pair-wise differences. There was a trend towards group differences for hip girth measurement, with the GTPS group having the highest mean girth (ANOVA: $p=0.090$, table 3 ). There were no group differences for waist girth and BMI measurements (table 3).

\section{Canonical discriminant analysis}

Using canonical discriminant analysis (CDA), the measures of adiposity and increased width at the superior greater trochanter were found to be relevant in discriminating across the four groups. The CDA had one out of three dimensions that was statistically significant ( $\mathrm{p}=0.048,0.458$ and 0.782 respectively; table S3). The statistically significant dimension had positive means for GTR (0.18) and GTPS (0.82), and negative means for OA $(-0.66)$ and ASC $(-0.68)$ (table S4). This dimension (dimension 1, table 4) represents gynoid adiposity, and width at the level of the superior GT, but not the lateral GT (table 4). The analysis confirms that overall adiposity, with a gynoid distribution is a factor in discriminating between the

Table 2 Bony morphology measures of the pelvis. Acetabular index, neck shaft angle, pelvic and greater trochanter widths. Results by group (mean, 95\% Cl)

\begin{tabular}{|c|c|c|c|c|c|}
\hline Measure & $\operatorname{ASC}(n=20)$ & GTPS $(n=28)$ & GTR $(n=21)$ & $O A(n=20)$ & ANOVA (p values) \\
\hline Neck-shaft angle ${ }^{\circ}$ & 137.2 (135.1 to 139.4$)$ & 137.1 (135.2 to 139.0$)$ & $133.1(131.0$ to 135.2$)$ & 137.0 (134.8 to 139.1$)$ & $0.021^{*}$ \\
\hline Acetabular index ${ }^{\circ}$ & 41.3 (39.8 to 42.9$)$ & 39.9 (38.6 to 41.3$)$ & 39.7 (38.2 to 41.3$)$ & 41.9 (40.3 to 43.5 ) & 0.129 \\
\hline Acetabular width $(\mathrm{A}: \mathrm{B})$ & $1.14(1.10$ to 1.17$)$ & 1.14 (1.08 to 1.20$)$ & $1.16(0.93$ to 1.40$)$ & $1.14(1.00$ to 1.27$)$ & 0.737 \\
\hline Superior GT width $(\mathrm{A}: \mathrm{C})$ & 0.87 (0.83 to 0.91$)$ & $0.91(0.89$ to 0.93$)$ & $0.90(0.0 .86$ to 0.94$)$ & $0.87(0.82$ to 0.92$)$ & 0.208 \\
\hline Lateral GT width (A:D) & $1.30(1.25$ to 1.35$)$ & $1.32(1.27$ to 1.37$)$ & 1.36 (1.29 to 1.43$)$ & $1.31(1.26$ to 1.36$)$ & 0.455 \\
\hline Superior to lateral GT (C:D) & 1.49 (1.44 to 1.54$)$ & 1.45 (1.41 to 1.49$)$ & $1.50(1.47$ to 1.53$)$ & $1.52(1.44$ to 1.60$)$ & 0.221 \\
\hline
\end{tabular}

ACS, asymptomatic control group; ANOVA, analysis of variance; GTPS, greater trochanteric pain syndrome group; GTR, gluteal tendon reconstruction group; OA, hip osteoarthritis group.

${ }^{*}$ Posthoc analysis found that the GTR group has a lower neck-shaft angle than the OA group $(p=0.012$ ), the GTPS group ( $p=0.005$ ) and the ASC group ( $p=0.007)$. No other group comparisons approached significance.

$\mathrm{A}$ is the distance from the midline to the AIIS. B is the distance from the midline to the lateral acetabulum. C is the distance from the midline to the most superior point of the greater trochanter. $\mathrm{D}$ is the distance from the midline to the most lateral aspect of the greater trochanter. 
Table 3 Results of anthropometric measurements. Group (mean, 95\% Cl)

\begin{tabular}{lccccc}
\hline Measurement & ASC $(\mathbf{n}=\mathbf{2 2})$ & GTPS $(\mathbf{n}=\mathbf{2 7})$ & GTR ( $\mathbf{n}=\mathbf{3 0})$ & OA (n=20) & ANOVA (p values) \\
\hline BMI (kgm $\left.{ }^{-2}\right)$ & $25.0(23.5$ to 26.5$)$ & $27.5(25.4$ to 29.6$)$ & $26.8(25.2$ to 28.4$)$ & $25.3(23.5$ to 27.1$)$ & 0.154 \\
Waist girth (cm) (W) & $83.0(78.9$ to 87.1$)$ & $88.4(82.9$ to 93.9$)$ & $84.6(79.6$ to 89.6$)$ & $84.0(79.0$ to 89.0$)$ & 0.420 \\
Hip girth (cm) (H) & $102.8(99.4$ to 106.2$)$ & $109.1(104.9$ to 113.2$)$ & $105.9(100.0$ to 109.2$)$ & $104.2(100.0$ to 108.4$)$ & 0.090 \\
GT girth (cm (GTg)) & $99.1(94.7$ to 103.5$)$ & $105.9(100.2$ to 111.6$)$ & $103.8(100.3$ to 107.3$)$ & $100.3(96.7$ to 103.9$)$ & $0.036^{*}$ \\
W:H & $0.81(0.783$ to 0.837$)$ & $0.79(0.753$ to 0.827$)$ & $0.80(0.764$ to 0.836$)$ & $0.81(0.781$ to 0.839$)$ & 0.884 \\
W:GTg & $0.85(0.791$ to 0.909$)$ & $0.84(0.797$ to 0.883$)$ & $0.82(0.772$ to 0.868$)$ & $0.84(0.791$ to 0.886$)$ & 0.855 \\
\hline
\end{tabular}

ACS, asymptomatic control group; ANOVA, analysis of variance; BMI, body mass index; GTg, greater trochanter girth; GTPS, greater trochanteric pain syndrome group; GTR, gluteal tendon reconstruction group; $\mathrm{H}$, hip girth; $\mathrm{OA}$, hip osteoarthritis group; $\mathrm{W}$, waist girth.

*Posthoc analysis showed a trend for the GTPS group to be larger about the greater trochanters than the ASC group, but pair wise analyses failed to demonstrate group differences, $p=0.079$. None of the other measures approached significance.

Table 4 Canonical (discriminant) loading

\begin{tabular}{lc}
\hline & Dimension 1 \\
\hline Adiposity measures & \\
BMI & $\mathbf{0 . 4 5}$ \\
Waist girth & 0.28 \\
Hip girth & $\mathbf{0 . 5 3}$ \\
GT girth & $\mathbf{0 . 5 0}$ \\
Waist girth to hip girth & -0.10 \\
Waist girth to GT girth & -0.01 \\
Bony measures & \\
NoF angle & -0.19 \\
Acetabulum width (A:B) & 0.08 \\
Superior GT width (A:C) & $\mathbf{0 . 3 4}$ \\
Lateral GT width (A:D)) & 0.14 \\
Superior to lateral GT (C:D) & -0.28
\end{tabular}

BMI, body mass index; GT, greater trochanter; NoF, neck of femur. Refer to text for descriptions of A-D. As the measures of adiposity and pelvic width at the GT are all positive with hip girth, superior GT girth and BMI loading the dimension the most (in bold). Dimension 1 was interpreted to be the combination of gluteofemoral adiposity and width of the superior GT.

groups. These results are interpreted to mean that gynoid adiposity combined with increased width at the level of the superior greater trochanter were associated with, and may be risk factors for, GTPS and GTR.

\section{DISCUSSION}

GTPS is a common condition in older women for which few risk factors have been identified. This study aimed to establish if features of bony morphology of the pelvis or hip constitute a risk for this condition. In addition, overall adiposity and a lower femoral neck shaft angle was evaluated as associated factors. We found that increased coxa vara was a risk factor for having gluteal tendon reconstruction surgery for refractory GTPS (table 2). Further, we found that higher levels of adiposity and increased width at the level of the superior greater trochanter were associated with both GTPS and GTR participants (tables 2 and 3 ).

Women who underwent gluteal tendon reconstructive surgery demonstrated lower neck shaft angles than women in the other groups (table 2). This novel finding may explain why some women have a more severe and persistent presentation of GTPS. Finite element modelling has shown that the iliotibial band applies more medial pressure over the greater trochanter in femurs with lower neck shaft angles than in those with higher neck shaft angles. ${ }^{27}$ Along with medial compression of the tendon against the greater trochanter, lateral pressure from the iliotibial band is likely to result in increased compression of the gluteus medius tendon, resulting in tendinopathy and eventual tendon rupture. ${ }^{36-38}$

Furthermore, the difference in biomechanics may contribute to the breakdown of the gluteal tendons via pathological loading of the tendon. The lower angle likely results in an increase in the load taken by the gluteus medius tendon as the lever arm is likely to be relatively shortened: we found no overall increase in width at the level of the greater trochanter, thus the resultant geometry supports this conclusion. This likely leads to increased extracellular matrix turnover that may result in tendinopathy. ${ }^{39}$ The combination of increased compression and load fits the tendinopathy model proposed by Cook and Purdam. ${ }^{40} 41$

The assessment of adiposity demonstrated that the women with GTPS and GTR had a trend towards larger girth measurements at the waist and hip, and were larger at the greater trochanter level. These trends support the previous findings that women with increased adiposity, particularly gynoid adiposity, are at risk of tendinopathy. ${ }^{12}$

The common assertion that women who develop GTPS have wider hips is supported by this study, albeit in an unexpected way. On univariate analysis, women with GTPS did not have a wider bony pelvis or hips than women without GTPS. Rather, in those women who went on to have surgery, the lower neck shaft angle combined with the increased gynoid adipose tissue results in the outward appearance of wider hips. Women with GTPS who did not go on to have surgery, and had a normal neck shaft angle also had higher levels of gynoid adiposity, thus would appear wider.

Higher levels of adiposity within the GTPS and GTR groups were confirmed by the canonical discriminant analysis. This analysis found that the GTPS and GTR groups had higher group means than either the ASC or OA groups on the dimension which reflects adiposity, with a higher influence of the gynoid than the central adiposity. Like Gaida et al who found gynoid adiposity had a stronger association with tendinopathy in women than central adiposity, ${ }^{12}$ our study found higher levels of gynoid adiposity in the GTPS and GTR groups than the other two groups - although pair-wise analysis failed to show group differences. As with Gaida et al, there was no difference between the groups with respect to BMI. BMI is known to be a simple but insensitive measure of adiposity. ${ }^{15}$ The results of this study, and those of Gaida et al, ${ }^{12}$ suggest that in addition to BMI, measures of adiposity distribution should be reported in musculoskeletal studies. ${ }^{42} 43$ We note that gynoid adipocyte accumulation and tendinopathy are associated in women in general and GTPS in particular. ${ }^{11} \mathrm{We}$ propose that there may be local adipokine and bioactive peptide activity that contributes to the development of tendinopathy. ${ }^{44}$ 
The mean neck shaft angle was larger for the entire population sample than has been previously reported $\left(125^{\circ}\right.$ to $\left.133^{\circ}\right) .{ }^{45-47}$ Differences in the mean neck shaft angle could be due to method variations. In the current study, the assessors used similar methods as previously reported ${ }^{45-49}$ and demonstrated good to excellent inter and intratester reliability. This study was undertaken on actual patients as opposed to previous studies that were completed on x-rays of, or directly from, anatomical or cadaveric specimens (with only one paper reporting reliability). ${ }^{48}$ This suggests that measure of neck shaft angle taken from specimens may not translate to the live patient. As neck shaft angle lowers with age ${ }^{48}$ cadaveric specimens are likely to have lower neck shaft angles than the general population. A further difference may be that the cohort in this study of all female pelves had a systematic bias for a lower neck shaft angle. A previous study noted that lower neck shaft angles are associated with higher levels of weight bearing, ${ }^{48}$ it is conceivable that the small sample is from a less active sample population than previous studies - which included measures from historic data. Regardless, people who underwent GTR have a lower neck shaft angle than the comparison groups (table 2).

The following limitations of this study are acknowledged. This sample population was relatively small and from one geographic location. Further research should address both these issues, thus determining a robust neck shaft angle cut off, or population-specific cut offs. A further limitation is the casecontrol design. The adiposity results would be stronger had a prospective study been undertaken. The results demonstrate that the OA group had a lower level of adiposity, and in a different distribution, than the other two symptomatic groups. This result is despite probably low levels of activity in all three symptomatic groups. This suggests that undertaking a longitudinal study to evaluate if adiposity is a modifiable risk factor (rather than an association) would be valuable.

Further research into the biomechanics of the hip and pelvis, compression of the tissues around the GT and the relationship of adiposity to tendinopathy should be pursued. As the hip acts in three dimensions, examining the biomechanics of the hip joint axis of movement, trochanteric height, neck shaft angle and femoral anteversion in people who develop GTPS, would assist in understanding the mechanism of aetiology, and direct mechanical treatments.

The results from this study provide a clinical indicator (a lower neck shaft angle) for patients who may fail to respond to non-operative treatment of GTPS. Researchers in the musculoskeletal area should include measures of central and peripheral adiposity in addition to BMI.

\section{CONCLUSIONS}

The development of GTPS is likely to be multifactorial in nature. Women with GTPS appear wider at the hips than women without GTPS due to increased levels of peripheral adiposity and, in some cases, lower femoral neck shaft angles, rather than differences in bony width of the pelvis or hips. We found that women with gluteal tendon tears and with a lower femoral neck shaft angle are more at risk of failure of conservative management than those with a normal neck shaft angle. We also found that increased gynoid adiposity and pelvic width at the level of the superior aspect of the greater trochanter was associated with GTPS, supporting previous findings that gynoid adiposity is associated with tendinopathy.

\section{What this study adds}

- The measure of a neck shaft angles of $<134^{\circ}$ identifies those at risk of failing conservative treatment.

- Confirmation of the findings that peripheral adiposity is associated with the development of tendinopathy in women.

- Wider greater trochanter (superior aspect) and increased adiposity combine to increase the risk of GTPS. This may be due to increased internal compression and shear combined with adiposity-related systemic effects.

Acknowledgements The authors gratefully acknowledge the assistance Dr lain Stewart, Dr David McKenna, Rose Varga, Dr Al Burns and Dr Damian Smith.

Funding Angela Fearon was supported by an Australian National University scholarship. Funding for imaging was provided from university funding. Jill Cook was the Australian centre for research into sports injury and its prevention, which is one of the International Research Centres for Prevention of Injury and Protection of Athlete Health supported by the International Olympic Committee (IOC).

\section{Competing interests None.}

Ethics approval ACT Health Human Research Ethics Committee; The Australian National University Human Research Ethics Committee.

Provenance and peer review Not commissioned; externally peer reviewed.

Data sharing statement The data set is held by Dr Angela Fearon (angie.fearon@hiphealth.ca).

\section{REFERENCES}

1. Fearon AM, Scarvell JM, Cook JL, et al. Does ultrasound correlate with surgical or histologic findings in greater trochanteric pain syndrome? A pilot study. Clin Orthop Relat Res 2010;468:1838-44.

2. Walsh MJ, Walton JR, Walsh NA. Surgical repair of the gluteal tendons: a report of 72 cases. J Arthroplasty 2011;26:1514-19.

3. Rolf C, Movin T. Etiology, histopathology, and outcome of surgery in achillodynia. Foot Ankle Int 1997; 18:565-9.

4. Riley G. The pathogenesis of tendinopathy. A molecular perspective. Rheumatology (Oxford) 2004:43:131-42.

5. Meeuwisse WH, Tyreman $\mathrm{H}$, Hagel B, et al. A dynamic model of etiology in sport injury: the recursive nature of risk and causation. Clin J Sport Med 2007; 17:215-19.

6. Almekinders LC, Temple JD. Etiology, diagnosis, and treatment of tendonitis: an analysis of the literature. Med Sci Sports Exerc 1998;30:1183-90.

7. Mokone GG, Gajjar M, September AV, et al. The guanine-thymine dinucleotide repeat polymorphism within the tenascin- $C$ gene is associated with achilles tendon injuries. Am J Sports Med 2005;33:1016-21.

8. Maffulli N, Wong J, Almekinders LC. Types and epidemiology of tendinopathy. Clin Sports Med 2003;22:675-92.

9. Rompe JD, Segal NA, Cacchio A, et al. Home training, local corticosteroid injection, or radial shock wave therapy for greater trochanter pain syndrome. Am J Sports Med 2009;37:1981-90.

10. Segal NA, Harvey W, Felson DT, et al. Leg-length inequality is not associated with greater trochanteric pain syndrome. Arthritis Res Ther 2008;10:R62.

11. Gaida JE, Ashe MC, Bass SL, et al. Is adiposity an under-recognized risk factor for tendinopathy? A systematic review. Arthritis Rheum 2009;61:840-9.

12. Gaida JE, Alfredson H, Kiss ZS, et al. Asymptomatic Achilles tendon pathology is associated with a central fat distribution in men and a peripheral fat distribution in women: a cross sectional study of 298 individuals. BMC Musculoskelet Disord 2010;11:41.

13. Lequesne $\mathbf{M}$, Djian $\mathrm{P}$, Vuillemin $\mathrm{V}$, et al. Prospective study of refractory greater trochanter pain syndrome. MRI findings of gluteal tendon tears seen at surgery. Clinical and MRI results of tendon repair. Joint Bone Spine 2008;75:458-64.

14. Woodley SJ, Nicholson HD, Livingstone V, et al. Lateral hip pain: findings from magnetic resonance imaging and clinical examination. J Orthop Sports Phys Ther 2008;38:313-28.

15. Dalton M, Cameron AJ, Zimmet PZ, et al. Waist circumference, waist-hip ratio and body mass index and their correlation with cardiovascular disease risk factors in Australian adults. J Intern Med 2003;254:555-63.

16. Renström AF. Mechanism, diagnosis, and treatment of running injuries. Instr Course Lect 1993;42:225-34. 
17. Anderson K, Strickland SM, Warren R. Hip and groin injuries in athletes. Am J Sports Med 2001;29:521-33.

18. Sim FH, Scott SG. Injuries of the pelvis and hip in athletes: anatomy and function. In: Nicholson JA, Hershman EB, eds. The Lower Extremity and Spine in Sports Medicine. St Louis: Mosby 1986:1119-69.

19. Kulund DN. Pain under the heel in runners. Va Med 1988;115:340-2.

20. Iorio $\mathbf{R}$, Healy WL, Warren $\mathrm{PD}$, et al. Lateral trochanteric pain following primary total hip arthroplasty. J Arthroplasty 2006;21:233-6.

21. Harvey WF, Yang M, Cooke TD, et al. Association of leg-length inequality with knee osteoarthritis: a cohort study. Ann Intern Med 2010;152:287-95.

22. Anderson TP. Trochanteric bursitis: diagnostic criteria and clinical significance. Arch Phys Med Rehabil 1958;39:617-22

23. Hogervorst T, Bouma HW, de Vos J. Evolution of the hip and pelvis. Acta Orthop Supp/ 2009;80:1-39.

24. Almekinders LC, Weinhold PS, Maffulli N. Compression etiology in tendinopathy. Clin Sports Med 2003;22:703-10.

25. Björnsson H, Norlin R, Knutsson A, et al. Fewer rotator cuff tears fifteen years after arthroscopic subacromial decompression. J Shoulder Elbow Surg 2010:19:111-15.

26. Gillard GC, Reilly HC, Bell-Booth PG, et al. The influence of mechanical forces on the glycosaminoglycan content of the rabbit flexor digitorum profundus tendon. Connect Tissue Res 1979:7:37-46.

27. Birnbaum K, Pandorf T. Finite element model of the proximal femur under consideration of the hip centralizing forces of the iliotibial tract. Clin Biomech (Bristol, Avon) 2011;26:58-64

28. Altman R, Alarcón G, Appelrouth D, et al. The American College of Rheumatology criteria for the classification and reporting of osteoarthritis of the hip. Arthritis Rheum 1991;34:505-14.

29. Ege Rasmussen KJ, Fanø N. Trochanteric bursitis. Treatment by corticosteroid injection. Scand J Rheumatol 1985;14:417-20.

30. Krout RM, Anderson TP. Trochanteric bursitis: management. Arch Phys Med Rehabil 1959;40:8-14.

31. Sharp IK. Acetabular dysplasia. The Journal of Bone and Joint Surgery, British Volume 1961;43:268-72

32. Yusuf S, Hawken S, Ounpuu S, et al. Obesity and the risk of myocardial infarction in 27,000 participants from 52 countries: a case-control study. Lancet 2005:366:1640-9.

33. Lemieux S, Prud'homme D, Bouchard C, et al. A single threshold value of waist girth identifies normal-weight and overweight subjects with excess visceral adipose tissue. Am J Clin Nutr 1996:64:685-93.

34. Kirkwood BR, Sterne JAC. Essential Medical Statistics. Second Edition. Carlton, Victoria, Australia: Blackwell 2003.
35. Lampropoulou S, Nowicky AV. Evaluation of the numeric rating scale for perception of effort during isometric elbow flexion exercise. Eur J Appl Physiol 2012:112:1167-75.

36. Slack C, Bradley G, Beaumont B, et al. Changes in the morphology and synthetic activity of cultured rat tail tendon. Cell Tissue Res 1986:245: 359-68.

37. Gillard GC, Merrilees MJ, Bell-Booth PG, et al. The proteoglycan content and the axial periodicity of collagen in tendon. Biochem J 1977;163:145-51.

38. Samiric T, Parkinson J, llic MZ, et al. Changes in the composition of the extracellular matrix in patellar tendinopathy. Matrix Bio/ 2009;28:230-6.

39. Bank RA, TeKoppele JM, Oostingh G, et al. Lysylhydroxylation and non-reducible crosslinking of human supraspinatus tendon collagen: changes with age and in chronic rotator cuff tendinitis. Ann Rheum Dis 1999;58:35-41.

40. Cook JL, Purdam CR. Is tendon pathology a continuum? A pathology model to explain the clinical presentation of load-induced tendinopathy. Br J Sports Med 2009;43:409-16.

41. Cook JL, Purdam C. Is compressive load a factor in the development of tendinopathy? Br J Sports Med 2012;46:163-8.

42. Mason C, Craig CL, Katzmarzyk PT. Influence of central and extremity circumferences on all-cause mortality in men and women. Obesity (Silver Spring) 2008;16:2690-5.

43. Pischon T, Boeing $\mathrm{H}$, Hoffmann $\mathrm{K}$, et al. General and abdominal adiposity and risk of death in Europe. N Engl J Med 2008;359:2105-20.

44. McNulty AL, Miller MR, O'Connor SK, et al. The effects of adipokines on cartilage and meniscus catabolism. Connect Tissue Res 2011:52:523-33.

45. Nordin S, Zulkifli 0, Faisham WI. Mechanical failure of Dynamic Hip Screw (DHS) fixation in intertrochanteric fracture of the femur. Med J Malaysia 2001;56(Suppl D): 12-17.

46. Hoaglund FT, Low WD. Anatomy of the femoral neck and head, with comparative data from Caucasians and Hong Kong Chinese. Clin Orthop Relat Res 1980;152:10-16.

47. Toogood PA, Skalak A, Cooperman DR. Proximal femoral anatomy in the normal human population. Clin Orthop Relat Res 2009;467:876-85.

48. Anderson JY, Trinkaus E. Patterns of sexual, bilateral and interpopulational variation in human femoral neck-shaft angles. J Anat 1998;192:279-85.

49. Richards CJ, Giannitsios D, Huk OL, et al. Risk of periprosthetic femoral neck fracture after hip resurfacing arthroplasty: valgus compared with anatomic alignment. A biomechanical and clinical analysis. J Bone Joint Surg Am 2008;90(Suppl 3):96-101. 